Check for updates

Cite this: RSC Adv., 2017, 7, 31069

\title{
Heat shock protein 90 and serine/threonine kinase B-Raf inhibitors have overlapping chemical space $\dagger$
}

Received 25th May 2017

Accepted 10th June 2017

DOI: 10.1039/c7ra05889f

rsc.li/rsc-advances

\author{
A. Anighoro, (D) ${ }^{a}$ L. Pinzi, ${ }^{b}$ G. Marverti, ${ }^{c}$ J. Bajorath (D) *a and G. Rastelli (D)*b
}

Heat shock protein 90 (Hsp90) and B-Raf are validated targets for anticancer drug discovery. Although there is strong evidence that concomitant inhibition of $\mathrm{Hsp90}$ and B-Raf may provide significant therapeutic benefits, molecules endowed with dual activity against the two targets have not been reported. For the first time, we show that Hsp90 and B-Raf inhibitors have overlapping chemical space and we disclose the first-in-class dual inhibitors. The compounds were identified through a computational strategy especially devised for detecting ligands with dual-target activity. Although the two targets had only remote binding site similarity, we were able to identify dual inhibitors with well-balanced in vitro potencies and relatively low molecular weight. Remarkably, they also inhibited the V600E mutant form of B-Raf with similar potency. This study provides the first direct proof that designing dual ligands of Hsp90 and a kinase is possible, thus opening the way to new interesting possibilities in drug discovery.

\section{Introduction}

Hsp90 and B-Raf are two established anticancer drug targets. Hsp90 is an ubiquitous molecular chaperone responsible for the assembly and regulation of many signal transduction and regulatory client proteins. ${ }^{1}$ Hsp90 refolds, stabilizes and regulates the trafficking of many proteins involved in uncontrolled proliferation and apoptotic resistance, including multiple protein kinases, steroid hormone receptors, mutated p53, survivin and others., ${ }^{1,2}$ Mapping of the interaction network of Hsp90 clearly demonstrated that this chaperone is a key node in multiple pathways and cellular and disease processes. ${ }^{3}$ Interestingly, many mutant and overexpressed oncoproteins are client proteins of Hsp90. ${ }^{\mathbf{1 , 2}}$ Unfortunately, despite the availability of a large arsenal of Hsp90 inhibitors belonging to different chemical classes, none of them has yet been approved as a drug, due to non-optimal safety profiles or lack of efficacy., ${ }^{\mathbf{4 , 5}}$ B-Raf is a member of the Ser/Thr protein kinase family involved in the MAPK signaling cascade, which plays a pivotal role in the regulation of many essential cell processes such as signal transduction, cell proliferation and cell survival. ${ }^{6}$ Activating mutations in B-Raf such as V600E bypass the regulation of MAPK signaling pathway and stimulate uncontrolled cell

${ }^{a}$ Department of Life Science Informatics, B-IT, LIMES Program Unit Chemical Biology and Medicinal Chemistry, Rheinische Friedrich-Wilhelms-Universität, Dahlmannstr. 2, D-53113 Bonn, Germany.E-mail: bajorath@bit.uni-bonn.de

${ }^{b}$ Department of Life Sciences, University of Modena and Reggio Emilia, Via Campi 103, 41125, Modena, Italy. E-mail: giulio.rastelli@unimore.it

${ }^{c}$ Department of Biomedical, Metabolic and Neurosciences, University of Modena and Reggio Emilia, Via Campi 287, 41125, Modena, Italy

$\dagger$ Electronic supplementary information (ESI) available. See DOI: $10.1039 / \mathrm{c} 7 \mathrm{ra05889f}$ proliferation. ${ }^{7}$ This poses a great challenge for therapeutic intervention because B-Raf mutations are associated with resistance to conventional chemotherapeutic drugs. Although a few B-Raf inhibitors have received approval for the treatment of certain types of cancer, responses are often temporary, rarely complete, and restricted by the onset of drug resistance. ${ }^{8}$ Therefore, there is a pressing need to develop new drugs with improved clinical profiles.

Notably, both B-Raf and B-RafV600E are client proteins of Hsp90. ${ }^{1,2}$ Therefore, inhibition of this chaperone would contribute to degradation of B-Raf and mutant B-Raf. ${ }^{9}$ Importantly, several recent studies demonstrated that combinations of various Hsp90 and B-Raf inhibitors provided significant synergistic effects. ${ }^{10-15}$ Indeed, drug combinations based on Hsp90 and B-Raf inhibitors (such as AT13387 and dabrafenib, or XL888 and vemurafenib) are currently under evaluation in clinical trials against mutated melanoma (http://ClinicalTrials.gov identifiers: NCT02721459, NCT02097225, NCT01657591). In addition, inhibition of Hsp90 may be effective in patients with intrinsic or acquired resistance to RAF inhibitors. ${ }^{10-15}$ Finally, B-Raf inhibitors may paradoxically activate the MAP kinase pathway, and inhibition of Hsp90 may help overcome such resistance mechanisms. ${ }^{16,17}$ Therefore, although drug development has been focused on targeting Hsp90 and B-Raf independently, considerable evidence has accumulated indicating that a combined inhibition of the two targets may be clinically relevant.

Despite the high therapeutic relevance of combination therapies, a single molecule with dual activity may show potential advantages, including but not limited to superior efficacy, a more predictable pharmacokinetic profile, and lower toxicity. ${ }^{18}$ In light of all these considerations, we reasoned that a multitarget drug, i.e. a small molecule that is able to hit multiple targets, should be 
of particular interest. This concept is in line with polypharmacology, which potentially limits drawbacks arising from the use of a single-target drug or a combination of multiple drugs. ${ }^{18-20}$ To the best of our knowledge, this possibility has not been explored in Hsp90 and B-Raf, and molecules endowed with dual activity are not available. Although the design of dual inhibitors of Hsp90 and B-Raf is hampered by the fact that the binding sites of the two targets significantly differ, we recently presented evidence from structural and computational analysis that inhibitors of Hsp90 and various kinases display at least remotely similar protein-ligand interaction patterns. ${ }^{21}$ These insights also motivated the search for dual inhibitors of Hsp90 and B-Raf. To these ends, a combined ligand- and structurebased virtual screening protocol was devised, which enabled the identification of the first-in-class compounds with dual activity. Remarkably, the compounds were also active against mutant B-RafV600E. This study provides the first evidence that Hsp90 and B-Raf inhibitors share a common chemical space, a finding that may open new avenues for future drug discovery.

\section{Results and discussion}

The analysis of available X-ray structures of Hsp90 and B-Raf in complex with various inhibitors (see Experimental and ESI

Table 1 X-ray structures used for virtual screening

\begin{tabular}{lllll}
\hline Protein & PDB code & Resolution $(\AA)$ & Ligand ID & Ki (nM) \\
\hline B-Raf & 3IDP & 2.70 & L1E & 1 \\
Hsp90 & 3RLR & 1.70 & 3RR & 30
\end{tabular}

sections $\dagger$ ) identified two active compounds containing a similar scaffold (Table 1, Fig. 1A and S1 $\dagger$ ) including 3RR (Hsp90 inhibitor) and L1E (B-Raf inhibitor). This scaffold was involved in well-defined interactions with both targets. Therefore, on the basis of visual inspection of the co-crystallized complexes of these inhibitors, a simple pharmacophore model was generated comprising a hydrogen bond donor, a hydrogen bond acceptor, and an aromatic center (Fig. 1B). The donor and acceptor formed hydrogen bonds with the hinge region of B-Raf and residues Asp93 and Thr184 of Hsp90. ${ }^{22,23}$ In addition, the aromatic feature centered on the phenyl ring was involved in hydrophobic contacts with both targets. Visual inspection of the two X-ray complexes suggested that the inclusion of pharmacophoric features derived from the larger ligand L1E that are not shared by 3RR would have probably resulted in large solvent-exposed moieties in Hsp90 structures. Therefore, such features were not included.

A substructure search was carried out to pre-select a subset of 15167 database compounds based on the Hsp90/B-Raf inhibitor scaffold shown in Fig. 1A. The obtained library of compounds was subjected to pharmacophore-guided docking to both targets, in order to prioritize compounds establishing the key interactions discussed above, leading to the selection of 20 candidates for dual inhibition, which were prioritized by comparing target-based docking ranks and visual inspection.

Evaluation of the candidate compounds in Hsp90 and B-Raf enzyme assays identified two dual inhibitors with micromolar activity against both targets. Fig. 2 shows the structures of the newly identified dual inhibitors, and their activities are reported in Table 2. Dose-response curves for these compounds are shown in Fig. S2 and S3 of the ESI. $\uparrow$ Both compounds contain a thieno[2,3- $d]$ pyrimidine scaffold and are distinguished by
A

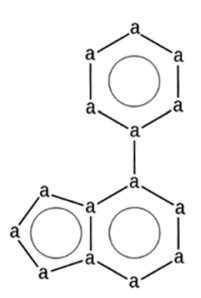

$\mathrm{a}=$ Any aromatic atom

C

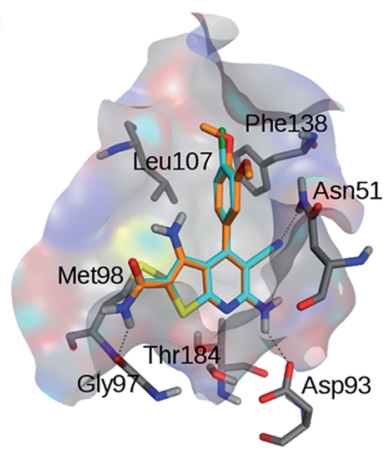

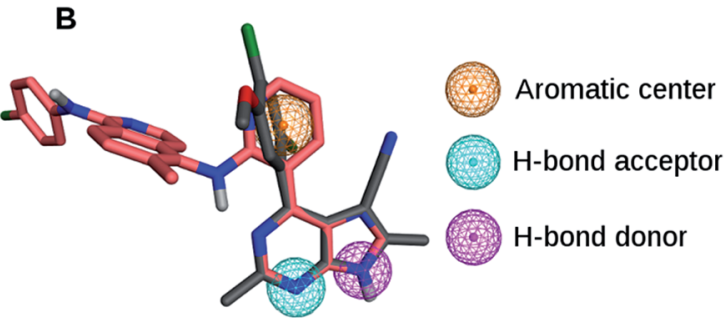

D

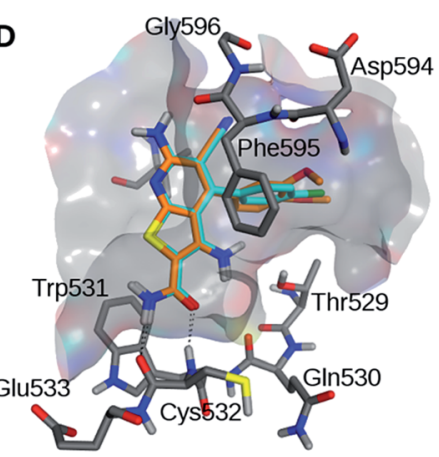

Fig. 1 Ligand alignments and pharmacophores. (A) Common scaffold identified for B-Raf and Hsp90 inhibitors in co-crystal structures 3 IDP and 3RLR. (B) Pharmacophore model based on the aligned B-Raf (pink) and the Hsp90 (gray) inhibitors. (C) Compounds 1 (cyan) and 2 (orange) docked to Hsp90. (D) Compounds 1 and 2 docked to B-Raf. 
1

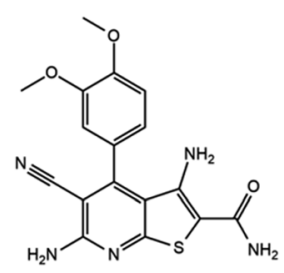

2

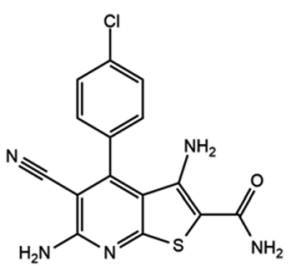

Fig. 2 Dual inhibitors of Hsp90 and B-Raf.

Table 2 Dual inhibitors of Hsp90 and B-Raf

\begin{tabular}{llll}
\hline Compound & IC $_{50}$ B-Raf $(\mu \mathrm{M})$ & IC $_{50}$ B-RafV600E $(\mu \mathrm{M})$ & IC $_{50} \operatorname{Hsp90}(\mu \mathrm{M})$ \\
\hline 1 & $28.9 \pm 1.7$ & $9.1 \pm 0.4$ & $1.2 \pm 0.1$ \\
2 & $1.5 \pm 0.1$ & $2.5 \pm 0.2$ & $7.6 \pm 0.6$
\end{tabular}

substitutions on the attached phenyl ring..$^{24,25}$ The compounds were also tested in an orthogonal assay to demonstrate that they did not elicit false-positive signals (Fig. S4†).

Putative binding modes of the dual inhibitors in Hsp90 and B-Raf are shown in Fig. 1C and D, respectively. In Hsp90, both compounds are likely to form a hydrogen bond network with Asn251, Asp93, and Thr184. The dimethoxyphenyl moiety of 1 and the corresponding chlorophenyl moiety of 2 are likely to form hydrophobic contacts with several residues including Leu107 and Phe138. This binding mode was consistent with the crystallographic structure of a Hsp90 inhibitor containing this scaffold (Fig. S5†). ${ }^{25}$

In B-Raf, the amide group of the inhibitors is thought to hydrogen bond to the hinge residue Cys532. In addition, the cyano group and the differently substituted phenyl ring are proximal to residues of the DFG motif.

The two inhibitors were also tested on the mutant BRafV600E, and were both found to be active with IC50 values comparable to, or better than, those obtained for wild type B-Raf (Table 2, Fig. S2 $\dagger$ ). These findings were considered particularly significant because B-RafV600E is a known escape mutant for therapeutic intervention.

Another advantage of the newly identified dual inhibitors is that they are small in size. We note that chimeras with dual activity are generally sought by merging two ligands with a suitable linker. This inevitably increases molecular weight, which may not only increase the likelihood of promiscuity, but also lead to poorer ADME/PK properties. ${ }^{20}$ By contrast, the newly identified inhibitors leave considerable room for further chemical optimization. For example, analysis of the predicted binding modes suggests that modifications at the phenyl ring of $\mathbf{1}$ and $\mathbf{2}$ may provide valuable opportunities for dual activity optimization. Larger groups are sterically tolerated and could provide additional van der Waals contacts with both targets. Moreover, polar substituents in meta- or para-position may provide additional hydrogen bonds with the backbone of Phe138 in Hsp90 and with Lys483 and Glu501 in B-Raf. These interactions have been observed in several Hsp90 and B-Raf crystal structures (e.g. PDB codes 1OSF and 2QG0 for Hsp90,

and 3D4Q, 3PPK, and 3OG7 for B-Raf). Moreover, the cyano group is solvent-exposed in B-Raf and is not required for Hsp90 activity, ${ }^{25}$ and could be replaced with other small hydrogen bonding groups. While chemical optimization is beyond the scope of the present work, the identified dual ligands constitute valuable starting points for further drug development.

\section{Conclusions}

In conclusion, we have reported on the identification of the first Hsp90 and B-Raf dual inhibitors and provided proof of concept that Hsp90 and B-Raf kinase inhibitors have overlapping chemical space. The identified compounds share a thieno[2,3- $d]$ pyrimidine scaffold that is also found in other Hsp90 and B-Raf inhibitors but has never been reported to represent compounds with dual activity. Interestingly, both targets belong to different families and adopt different folds. Binding site similarities estimated with a method particularly efficient in detecting binding site similarity in the absence of sequence or fold conservation confirmed that Hsp90 and B-Raf binding sites significantly differ (see Experimental section for details). Thus, demonstration of dual inhibition properties was far from being obvious.

Remarkably, both compounds displayed similar activity against mutant B-RafV600E, which is of considerable potential for cancer treatment.

If synergy exists between two targets, as is the case for Hsp90 and B-Raf, ${ }^{9-15}$ it is conceivable that dual inhibitors with only moderate activity might still produce superior pharmacological effects in terms of efficacy and safety as compared with highly potent compounds that only inhibit a single target. ${ }^{26,27}$ Remarkably, the discovered compounds displayed a balanced level of activity among Hsp90, B-Raf, and mutant B-Raf, which makes them especially promising in a polypharmacology approach context ${ }^{18,26,27}$ More in general, this study provides the first direct proof that designing dual ligands of Hsp90 and a kinase is possible, thus opening the way to new interesting possibilities in drug discovery based on rational polypharmacology approaches.

\section{Experimental section}

\section{$\mathrm{X}$-ray structures and pharmacophore model}

For B-Raf, eight crystal structures in complex with potent inhibitors were selected for which high confidence activity data were available (Table S1†). ${ }^{28}$ In addition, for Hsp90, 19 structures of inhibitor complexes with Ki values of less than $10 \mu \mathrm{M}$ were selected (Table S1 $\dagger$ ). Analysis of these structures identified two inhibitors with a structurally related scaffold for B-Raf with Protein Data Bank (PDB) ${ }^{29}$ code 3 IDP $^{22}$ and Hsp90 with PDB code $3 R_{L}{ }^{23}$ (Fig. 1A). The bound conformations of these inhibitors (termed L1E and 3RR) were superposed and a basic pharmacophore model was derived using the Molecular Operating Environment 2014.09 $(\mathrm{MOE})^{30}$ that included a hydrogen bond donor, a hydrogen bond acceptor, and an aromatic center (Fig. 1B). These features were likely to engage in key interactions with both targets. ${ }^{22,23}$ 


\section{Compound database and pre-filtering}

A database of 4805970 unique compounds was obtained by merging the catalogs of 10 vendors (including AMRI, Aronis, Asinex, ChemBridge, Enamine, InterBioScreen, Life Chemicals, Maybridge, Otava and Vitas-MLab). A SMARTS string representing the shared scaffold (Fig. 1A) was used to filter the database with OpenBabel 2.3.2 (ref. 31) that permitted possible heteroatoms and substitutions preserving the aromatic character of the rings (Fig. 1A). On the basis of this search, 15167 compounds were pre-selected and prepared for docking with MOE by computing protonation states and partial charges and optimizing structures by energy minimization.

\section{Protein structure preparation}

In X-ray structure 3IDP, 15 residues (residues from 598 to 613) of the activation loop were missing. ${ }^{22}$ An initial conformation of this loop was obtained by adding the missing residues with MOE followed by relaxation of the loop structure and refinement with ModLoop. ${ }^{32}$ This tool generates 300 loop conformations and returns the one with the most favorable energy score. The 3IDP and 3RLR template structures were prepared for docking with MOE by adding hydrogen atoms and calculating atomic partial charges according to the MMFF94x force field. All water molecules were removed except for one water molecule in Hsp90 (number 2 according to 3RLR residues numbering) that was known to be involved in a conserved network of hydrogen bonds between Hsp90 and several inhibitors. ${ }^{23,33,34}$

\section{Docking}

Docking calculations on Hsp90 and B-Raf binding were performed with the Dock module of $\mathrm{MOE}{ }^{30}$ The binding sites were defined by the coordinates of the co-crystallized ligands. The pharmacophore model (Fig. 1B) was used to guide docking of the 15167 pre-selected compounds. In each case, 1000 docking poses were generated with the pharmacophore placement function of MOE. Docking poses were ranked on the basis of force field scoring and for each ligand, the top 10 poses were selected and subjected to energy refinement and re-scoring. Ultimately, the best-scoring poses were ranked and retained if they matched the pharmacophore model in a post-filtering step. For both targets, highly ranked compounds were visually inspected to aid in the final selection of 20 candidate compounds for experimental evaluation. Test compounds were also computationally screened for pan assay interference compounds (PAINS) ${ }^{35}$ using publicly available filters ${ }^{36}$ and aggregating compounds using the ZINC/UCSF aggregator advisor. ${ }^{37}$

\section{Enzyme assays}

Candidate compounds were assayed for in vitro inhibitory activity against both targets.

B-Raf. B-Raf assays were performed at BPS, San Diego, using the Kinase-Glo Plus luminescence kinase assay test system (Promega). ${ }^{38,39}$ Selected compounds were first dissolved in $10 \%$ DMSO, then $5 \mu \mathrm{l}$ of the resulting solution was added to $50 \mu \mathrm{l}$ of a reaction mixture containing $40 \mathrm{mM}$ Tris $\mathrm{pH} 7.4,10 \mathrm{mM}$ $\mathrm{MgCl}_{2}, 0.1 \mathrm{mg} \mathrm{ml}^{-1} \mathrm{BSA}, 1 \mathrm{mM}$ DTT, $0.1 \mathrm{mg} \mathrm{ml}^{-1}$ inactive MEK1 substrate, $10 \mu \mathrm{M}$ ATP and B-Raf kinase. A final concentration of $1 \%$ DMSO was obtained in all solutions. All enzymatic reactions were conducted at $\mathrm{pH} 7.4$ and $30{ }^{\circ} \mathrm{C}$ for 40 minutes. After the enzymatic reaction, $50 \mu \mathrm{l}$ of the Promega kit was added to each reaction and plates were incubated for 5 minutes at room temperature. Finally, luminescent signals were measured using a BioTek Synergy 2 microplate reader. Assays were performed in duplicate at each concentration. The luminescence data were analyzed using the computer software Graphpad Prism and $\mathrm{IC}_{50}$ values and titration curves were evaluated (Table 2, Fig. S2 $\dagger$ ). Vemurafenib was used as a control B-raf inhibitor. ${ }^{40}$ The same protocol was used to determine the inhibitory activity against the B-RafV600E mutant.

Hsp90. Hsp90 assays were performed at Nerviano Medical Sciences (NMS) using a displacement fluorescence polarization technique. ${ }^{41}$ Recombinant HSP90 $\alpha$ preparations were $>80 \%$ pure on the basis of SDS-PAGE, N-terminal sequence analysis, and electrospray mass spectrometry. Candidate compounds were first solubilized at $10 \mathrm{mM}$ in $100 \%$ DMSO, then serially diluted 1 to 3 (10 concentrations) in duplicate in 384 well plates. Afterwards they were directly diluted in the reaction mixture in order to obtain compound concentrations ranging from 0.005 to $100 \mu \mathrm{M}$ and a final DMSO concentration of $1 \%$. An Hsp90 $\alpha$ binding probe (HBP) was synthesized by coupling an ATPcompetitive standard compound to the fluorophore Atto-610 (Atto-Tec GmbH, Germany). HBP bound to the N-terminal ATP binding site, as proven by complete displacement by reference inhibitors BIIB021, geldanamycin and CCT018159. ${ }^{42,43}$ Using a Beckman NX instrument, $19 \mu \mathrm{l}$ of the reaction mix (50 $\mathrm{nM} \mathrm{Hsp} 90 \alpha$ protein and $50 \mathrm{nM}$ HBP final concentration) were dispensed in 384-well black plates (with no binding surface, Corning 3575). Protein and probe were diluted in $50 \mathrm{mM}$ Hepes $\mathrm{pH}$ 7, $100 \mathrm{mM} \mathrm{KCl,} 5 \mathrm{mM} \mathrm{MgCl} 2,1 \mathrm{mM}$ EDTA, $0.001 \%$ Triton. For negative (low control, LC) and positive (high control, HC) controls, HBP alone or Hsp90 $\alpha+$ HBP were dispensed in the well, respectively. The compounds in $100 \%$ DMSO at a volume of $0.19 \mu \mathrm{l}$ were dispensed in the working plate using a pin-tool head. For LC and HC, only DMSO was added to a final concentration of 1\%. A SAFIRE2 (TECAN) microreader was used to read the plates (Ex $590 \mathrm{~nm}$ and Em $630 \mathrm{nM}, G$ factor calculated with negative control was 0.854). For $\mathrm{IC}_{50}$ determination, all data were analyzed using the program Prism5, which provides sigmoidal fittings of the doseresponse curves using a four-parameter logistic equation. Results are reported in Table 2 and Fig. S3.† BIIB021 was used as a reference inhibitor. ${ }^{42}$

\section{Orthogonal assay}

To test for potential false-positive Hsp90 and/or B-Raf assay readouts as a consequence of compound aggregation, an orthogonal enzyme assay was carried out at NMS focusing on inhibition of Poly(ADP-Ribose)-Polymerase 1 (PARP-1). Recombinant PARP-1 preparations were $>80 \%$ pure on the basis of SDSPAGE, N-terminal sequence analysis and electrospray mass 
spectrometry. A fluorescence polarization assay was conducted to evaluate the displacement of a PARP-1 Binding Probe (PBP) synthesized as described previously. ${ }^{44}$ The $\mathrm{NAD}^{+}$competitive PARP-1 inhibitor olaparib was used as a control. ${ }^{45}$ The compounds were solubilized, serially diluted and dispensed in 384-well black plates by following the same procedure described for the Hsp90 assays. $19 \mu \mathrm{l}$ of the reaction mixture (final concentration of $250 \mathrm{nM}$ PARP-1 protein and $50 \mathrm{nM}$ PBP) were dispensed in 384-well black plate. Protein and probe were diluted in $50 \mathrm{mM}$ Tris/HCl pH 8, $150 \mathrm{mM} \mathrm{NaCl}, 10 \mathrm{mM} \mathrm{MgCl}_{2}, 0.001 \%$ Triton. Plate reading ( $G$ factor calculated with negative control was 1.160) and $\mathrm{IC}_{50}$ determinations were then performed as described for Hsp90. Results are shown in Fig. S4. $\dagger$

\section{Binding site similarity calculations}

Hsp90 and B-Raf binding sites were compared using Volsite and Shaper. ${ }^{46}$ Crystal structures of human B-Raf and Hsp90 were collected from the PDB (accessed on February 2017) ${ }^{29}$ and chains were split, leading to two final sets of 119 and 244 structures for B-Raf and Hsp90, respectively. For each set, structures were aligned with the Align Binding Sites tool available in Maestro (version 10.3) ${ }^{47}$ and a representative structure for each significantly different conformation was selected, obtaining a final set of 9 representative conformations for B-Raf and 11 for Hsp90. The proteins were prepared with the Protein Preparation Wizard utility of Maestro 10.3. Binding sites were then described with VolSite and compared with Shaper, using default settings. ${ }^{\mathbf{4 6}}$ All ligand and water molecules were removed from the representative structures, except for water molecules involved in a conserved network of hydrogen bonds between Hsp90 and the co-crystallized inhibitors. ${ }^{34}$ An average RefTversky similarity score of 0.33 was obtained, which was below the statistical threshold of 0.35 that distinguishes similar from dissimilar binding sites. ${ }^{46}$

\section{Conflicts of interest}

The authors declare no competing financial interest.

\section{Notes and references}

1 J. Li and J. Buchner, J. Biomed. Sci., 2013, 36, 106-117.

2 P. C. Echeverría and D. Picard, in The Molecular Chaperones Interaction Networks in Protein Folding and Degradation, ed. W. A. Houry, Springer-Verlag, New York, 2014, ch. 5, pp. 133-150.

3 P. C. Echeverría, A. Bernthaler, P. Dupuis, B. Mayer and D. Picard, PLoS One, 2011, 6, e26044.

4 L. Neckers and P. Workman, Clin. Cancer Res., 2012, 18, 6476.

5 R. Bhat, S. R. Tummalapalli and D. P. Rotella, J. Med. Chem., 2014, 57, 8718-8728.

6 W. Zhang and H. T. Liu, Cell Res., 2002, 12, 9-18.

7 H. Davies, G. R. Bignell, C. Cox, P. Stephens, S. Edkins, S. Clegg, J. Teague, H. Woffendin, M. J. Garnett, W. Bottomley, N. Davis, E. Dicks, R. Ewing, Y. Floyd,
K. Gray, S. Hall, R. Hawes, J. Hughes, V. Kosmidou, A. Menzies, C. Mould, A. Parker, C. Stevens, S. Watt, S. Hooper, R. Wilson, H. Jayatilake, B. A. Gusterson, C. Cooper, J. Shipley, D. Hargrave, K. Pritchard-Jones, N. Maitland, G. Chenevix-Trench, G. J. Riggins, D. D. Bigner, G. Palmieri, A. Cossu, A. Flanagan, A. Nicholson, J. W. C. Ho, S. Y. Leung, S. T. Yuen, B. L. Weber, H. F. Seigler, T. L. Darrow, H. Paterson, R. Marais, C. J. Marshall, R. Wooster, M. R. Stratton and P. A. Futreal, Nature, 2002, 417, 949-954.

8 H. E. Haarberg and K. S. M. Smalley, Drug Discovery Today: Technol., 2014, 11, 27-32.

9 O. M. Grbovic, A. D. Basso, A. Sawai, Q. Ye, P. Friedlander, D. Solit and N. Rosen, Proc. Natl. Acad. Sci. U. S. A., 2006, 103, 57-62.

10 K. H. T. Paraiso, H. E. Haarberg, E. Wood, V. W. Rebecca, Y. A. Chen, Y. Xiang, A. Ribas, R. S. Lo, J. S. Weber, V. K. Sondak, J. K. John, A. A. Sarnaik, J. M. Koomen and K. S. M. Smalley, Clin. Cancer Res., 2012, 18, 2502-2514.

11 Z. Solárová, J. Mojžiš and P. Solár, Int. J. Oncol., 2015, 46, 907-926.

12 J. Acquaviva, D. L. Smith, J.-P. Jimenez, C. Zhang, M. Sequeira, S. He, J. Sang, R. C. Bates and D. A. Proia, Mol. Cancer Ther., 2014, 13, 353-363.

13 G. Zhang, D. T. Frederick, L. Wu, Z. Wei, C. Krepler, S. Srinivasan, Y. C. Chae, X. Xu, H. Choi, E. Dimwamwa, O. Ope, B. Shannan, D. Basu, D. Zhang, M. Guha, M. Xiao, S. Randell, K. Sproesser, W. Xu, J. Liu, G. C. Karakousis, L. M. Schuchter, T. C. Gangadhar, R. K. Amaravadi, M. Gu, C. Xu, A. Ghosh, W. Xu, T. Tian, J. Zhang, S. Zha, Q. Liu, P. Brafford, A. Weeraratna, M. A. Davies, J. A. Wargo, N. G. Avadhani, Y. Lu, G. B. Mills, D. C. Altieri, K. T. Flaherty and M. Herlyn, J. Clin. Invest., 2016, 126, 1834-1856.

14 R. Suzuki, S. Kikuchi, T. Harada, N. Mimura, J. Minami, H. Ohguchi, Y. Yoshida, M. Sagawa, G. Gorgun, D. Cirstea, F. Cottini, J. Jakubikova, Y.-T. Tai, D. Chauhan, P. G. Richardson, N. Munshi, K. Ando, T. Utsugi, T. Hideshima and K. C. Anderson, PLoS One, 2015, 10, e0143847.

15 T. Smyth, K. H. T. Paraiso, K. Hearn, A. M. Rodriguez-Lopez, J. M. Munck, H. E. Haarberg, V. K. Sondak, N. T. Thompson, M. Azab, J. F. Lyons, K. S. M. Smalley and N. G. Wallis, Mol. Cancer Ther., 2014, 13, 2793-2804.

16 P. I. Poulikakos, C. Zhang, G. Bollag, K. M. Shokat and N. Rosen, Nature, 2010, 464, 427-430.

17 M. Phadke, G. T. Gibney, C. J. Rich, I. V. Fedorenko, Y. A. Chen, R. R. Kudchadkar, V. K. Sondak, J. Weber, J. L. Messina and K. S. M. Smalley, J. Invest. Dermatol., 2015, 135, 2542-2544.

18 A. Anighoro, J. Bajorath and G. Rastelli, J. Med. Chem., 2014, 57, 7874-7887.

19 G. Rastelli and L. Pinzi, Front. Pharmacol., 2015, 6, 157.

20 J.-U. Peters, J. Med. Chem., 2013, 56, 8955-8971.

21 A. Anighoro, D. Stumpfe, K. Heikamp, K. Beebe, L. M. Neckers, J. Bajorath and G. Rastelli, J. Chem. Inf. Model., 2015, 55, 676-686. 
22 A. L. Smith, F. F. DeMorin, N. A. Paras, Q. Huang, J. K. Petkus, E. M. Doherty, T. Nixey, J. L. Kim, D. A. Whittington, L. F. Epstein, M. R. Lee, M. J. Rose, C. Babij, M. Fernando, K. Hess, Q. Le, P. Beltran and J. Carnahan, J. Med. Chem., 2009, 52, 6189-6192.

23 P.-P. Kung, P.-J. Sinnema, P. Richardson, M. J. Hickey, K. S. Gajiwala, F. Wang, B. Huang, G. McClellan, J. Wang, K. Maegley, S. Bergqvist, P. P. Mehta and R. Kania, Bioorg. Med. Chem. Lett., 2011, 21, 3557-3562.

24 G. K. Packard, P. Papa, J. R. Riggs, P. Erdman, L. Tehrani, D. Robinson, R. Harris, G. Shevlin, S. Perrin-Ninkovic, R. Hilgraf, M. A. McCarrick, T. Tran, Y. Fleming, A. Bai, S. Richardson, J. Katz, Y. Tang, J. Leisten, M. Moghaddam, B. Cathers, D. Zhu and S. Sakata, Bioorg. Med. Chem. Lett., 2012, 22, 747-752.

25 P. A. Brough, X. Barril, J. Borgognoni, P. Chene, N. G. M. Davies, B. Davis, M. J. Drysdale, B. Dymock, S. A. Eccles, C. Garcia-Echeverria, C. Fromont, A. Hayes, R. E. Hubbard, A. M. Jordan, M. R. Jensen, A. Massey, A. Merrett, A. Padfield, R. Parsons, T. Radimerski, F. I. Raynaud, A. Robertson, S. D. Roughley, J. Schoepfer, H. Simmonite, S. Y. Sharp, A. Surgenor, M. Valenti, S. Walls, P. Webb, M. Wood, P. Workman and L. Wright, J. Med. Chem., 2009, 52, 4794-4809.

26 R. Morphy and Z. Rankovic, Drug Discovery Today, 2007, 12, 156-160.

27 G. Bottegoni, A. D. Favia, M. Recanatini and A. Cavalli, Drug Discovery Today, 2012, 17, 23-34.

28 N. Furtmann, Y. Hu and J. Bajorath, J. Med. Chem., 2015, 58, 252-264.

29 H. M. Berman, J. Westbrook, Z. Feng, G. Gilliland, T. N. Bhat, H. Weissig, I. N. Shindyalov and P. E. Bourne, Nucleic Acids Res., 2000, 28, 235-242.

30 Molecular Operating Environment, version 2014.09, Chemical Computing Group, Inc.

31 N. M. O'Boyle, M. Banck, C. A. James, C. Morley, T. Vandermeersch and G. R. Hutchison, J. Cheminf., 2011, $3,33$.

32 A. Fiser and A. Sali, Bioinformatics, 2003, 19, 2500-2501.

33 N. G. M. Davies, H. Browne, B. Davis, M. J. Drysdale, N. Foloppe, S. Geoffrey, B. Gibbons, T. Hart, R. Hubbard, M. R. Jensen, H. Mansell, A. Massey, N. Matassova,
J. D. Moore, J. Murray, R. Pratt, S. Ray, A. Robertson, S. D. Roughley, J. Schoepfer, K. Scriven, H. Simmonite, S. Stokes, A. Surgenor, P. Webb, M. Wood, L. Wright and P. Brough, Bioorg. Med. Chem., 2012, 20, 6770-6789.

34 M. Sgobba and G. Rastelli, ChemMedChem, 2009, 4, 13991409.

35 J. Baell and M. A. Walters, Nature, 2014, 513, 481-483.

36 ZINC PAINS patterns search http://zinc15.docking.org/ patterns/home/(accessed June 17, 2016).

37 J. J. Irwin, D. Duan, H. Torosyan, A. K. Doak, K. T. Ziebart, T. Sterling, G. Tumanian and B. K. Shoichet, J. Med. Chem., 2015, 58, 7076-7087.

38 K. E. Mercer and C. A. Pritchard, Biochim. Biophys. Acta, 2003, 1653, 25-40.

39 C. K. Weber, J. R. Slupsky, H. A. Kalmes and U. R. Rapp, Cancer Res., 2001, 61, 3595-3598.

40 Y. Heakal, M. Kester and S. Savage, Ann. Pharmacother., 2011, 45, 1399-1405.

41 X. Huang, J. Biomol. Screening, 2003, 8, 34-38.

42 K. Lundgren, H. Zhang, J. Brekken, N. Huser, R. E. Powell, N. Timple, D. J. Busch, L. Neely, J. L. Sensintaffar, Y. Yang, A. McKenzie, J. Friedman, R. Scannevin, A. Kamal, K. Hong, S. R. Kasibhatla, M. F. Boehm and F. J. Burrows, Mol. Cancer Ther., 2009, 8, 921-929.

43 R. Howes, X. Barril, B. W. Dymock, K. Grant, C. J. Northfield, A. G. S. Robertson, A. Surgenor, J. Wayne, L. Wright, K. James, T. Matthews, K.-M. Cheung, E. McDonald, P. Workman and M. J. Drysdale, Anal. Biochem., 2006, 350, 202-213.

44 G. Papeo, N. Avanzi, S. Bettoni, A. Leone, M. Paolucci,

R. Perego, F. Quartieri, F. Riccardi-Sirtori, S. Thieffine, A. Montagnoli and R. Lupi, J. Biomol. Screening, 2014, 19, 1212-1219.

45 K. A. Menear, C. Adcock, R. Boulter, X. Cockcroft, L. Copsey, A. Cranston, K. J. Dillon, J. Drzewiecki, S. Garman, S. Gomez, H. Javaid, F. Kerrigan, C. Knights, A. Lau, V. M. Loh, I. T. W. Matthews, S. Moore, M. J. O'Connor, G. C. M. Smith and N. M. B. Martin, J. Med. Chem., 2008, 51, 6581-6591.

46 J. Desaphy, K. Azdimousa, E. Kellenberger and D. Rognan, J. Chem. Inf. Model., 2012, 52, 2287-2299.

47 Maestro, version 10.3 2015, Schrödinger, LLC, New York, NY. 